\title{
Efficacy of Jianpi Huatan Dingxuan Decoction in the Treatment of Vertigo and Phlegm Obstruction Syndrome
}

\author{
Weifeng Zheng ${ }^{\mathrm{a}, 1}$, Wentao Zhang ${ }^{1}$, Yuqin Wang ${ }^{1}$, Yinglin Cui*,1 (Corresponding Author) \\ ${ }^{1}$ Henan Provincial Hospital of Traditional Chinese Medicine Zhengzhou, Henan 450002 info@hnszyy.com.cn
}

\begin{abstract}
Objective: To explore the clinical effect of the application of Jianpi Huatan Dingxuan Decoction in the treatment of patients with vertigo and phlegm obstruction. Method: The research work was started in Henan Provincial Hospital of Traditional Chinese Medicine. The work will be carried out from October 2019 to October 2020. The patients are all patients with vertigo and phlegm obstruction syndrome who received treatment during this period. 100 of them were selected as this time. The study subjects were randomly divided into two groups, the control group given conventional western medicine treatment and the experimental group given Jianpi Huatan Dingxuan Decoction. The treatment effects of the two groups were compared and analyzed. Results: The treatment efficiency and nursing satisfaction of the experimental group were $96.00 \%$ and $94.00 \%$ respectively, which were significantly higher than the $82.00 \%$ and $78.00 \%$ of the control group. Before treatment, the vertebrobasilar blood flow velocity of the patients was relatively low, $\mathrm{P}>0.05$. After the treatment intervention, the vertebral artery blood flow velocity and the basilar artery blood flow velocity of the experimental group were significantly higher than those of the control group. The data differed greatly. $\mathrm{P}<0.05$, the experimental group had better results. Conclusion: The application of Jianpi Huatan Dingxuan Decoction in the treatment of patients with vertigo and phlegm obstruction has a significant effect, which can promote the recovery of patients, improve the effective rate of clinical treatment, and has positive significance for clinical development.
\end{abstract}

\section{Introduction}

Vertigo is quite common in clinical practice. Patients often show dizziness and it stops when they close their eyes. For patients with more serious conditions, it can even manifest as a situation such as traveling in a car or a boat, and the patient is accompanied by nausea, vomiting and sweating. Some patients may even faint at any time. From the perspective of western medicine, there are relatively many factors that cause patients to experience dizziness. For example, posterior cerebral circulation ischemia, Meniere's disease, etc., which can cause dizziness in patients. Patients are normally treated with medications ${ }^{[1]}$. On this basis, this study applied the Jianpi Huatan Dingxuan Decoction to explore its clinical application effects, and the related analysis is as follows.

\section{Purpose}

This research is through grouping and contrasting treatment, to explore the clinical value of applying Jianpi Huatan Dingxuan Decoction in patients with vertigo and phlegm obstruction syndrome, so as to provide a basis for clinical treatment.

\section{Method}

\subsection{Patient information}

This research work was selected to be carried out in our hospital, and the time was from February 2018 to March 2020. The patients were all patients with vertigo who were treated in our hospital during this period. The patients were in compliance with the "Diagnosis and Efficacy Standards of TCM Diseases", Manifested as typical dizziness symptoms: such as dizziness, sense of rotation, accompanied by nausea, vomiting, sweating, pale complexion and other situations. TCM syndrome differentiation is phlegm obstruction syndrome, manifested as: chest tightness, nausea, dizziness, head is heavy as wrapped, white and greasy tongue, slippery string. In this study, 100 patients were selected, in order to explore effective clinical treatment methods, they were randomly divided into two groups. One group was treated with conventional western medicine, which was the control group, and the other group was treated with Jianpi Huatan Dingxuan Decoction, which was the experiment group. The number of male and female patients in the experimental group were 31 and 19 respectively. The age of the patients was selected from 40 to 77 years old, and the average age was $(56.45 \pm 4.34)$ 
years. In the control group, the number of male and female patients were 32 and 18 respectively. The oldest patient was 78 years old and the youngest was 38 years old. The average age was $(55.44 \pm 4.56)$ years old. There was no significant difference in general information between the two groups, which means it is comparable.

\subsection{Application method}

In this study, patients in the control group were treated with conventional western medicine. During the specific treatment process, vertigo stopping medicine was applied at a dose of $25 \mathrm{mg}$, and nimodipine was used at a dose of $20 \mathrm{mg}$, taken orally 3 times a day, while buflomedil was used at a dose of $0.1 \mathrm{~g}$, in combination with $250 \mathrm{ml}$ of normal saline, and intervene by intravenous drip, once a day.

Patients in the experimental group were treated with Jianpi Huatan Dingxuan Decoction. In the specific implementation process, the drugs mainly included $10 \mathrm{~g}$ each of Gastrodia tuber, Pinellia ternata, and Inula, $15 \mathrm{~g}$ of Poria, $12 \mathrm{~g}$ of Atractylodes, $30 \mathrm{~g}$ each of Alisma and Ochre. If the patient has hyperactivity of the liver-yang, manifests as headache and swelling, easy irritation, and dizziness when angry, it need to increase $15 \mathrm{~g}$ of Uncaria, $15 \mathrm{~g}$ of Shijue Ming, and 12g of Radix Paeoniae Alba; If the patient has a deficiency of qi and blood, and the patient shows fatigue and laziness, it needs to increase $20 \mathrm{~g}$ of astragalus, $15 \mathrm{~g}$ codonopsis, $15 \mathrm{~g}$ of angelica; If the patient's kidney essence is insufficient and the waist is sore and the knees are soft, you need to add $15 \mathrm{~g}$ of cornus, $15 \mathrm{~g}$ of wolfberry, and $15 \mathrm{~g}$ of jujube seed; If the patient has congestion and obstructive orifice syndrome, the face and lips are dark and the tongue has ecchymosis, it need to add $10 \mathrm{~g}$ of Chuanqiang, $10 \mathrm{~g}$ of peach kernel, $10 \mathrm{~g}$ of safflower, 1 dose a day, decoction with water 2 times, in morning and evening.

\subsection{Observation indicators}

Clinical curative effect, including marked effective, effective and ineffective, marked effective means the patient's dizziness, neck and shoulder pain disappeared, effective means the patient's dizziness and neck pain and other clinical symptoms were improved, and ineffective means the patient's clinical symptoms did not improve before and after treatment, and even got aggravated. Excluding ineffective rate is the total effective rate of this study.

Vertebral-basal artery blood flow velocity measurement: Transcranial Doppler ultrasound examination is given to the patient before and after treatment. The patient is mainly given a sitting position to measure the Doppler movement signal of the vertebral artery and basilar artery, and its blood flow. The average speed is calculated.

The satisfaction degree of patients is compared, mainly including satisfactory, relatively satisfactory and unsatisfactory. A satisfaction survey questionnaire is used. A questionnaire score of 90 points or more is satisfactory, a score of 70-90 points is relatively satisfactory, and a score of 70 points or less is unsatisfactory, total satisfaction degree is the sum of satisfactory and relatively satisfactory.

\subsection{Statistical methods}

Perform statistics on the data and apply the software SPSS20.0. The analysis of measurement data $( \pm \mathrm{s})$ is verified by $\mathrm{t}$ value, while the calculation and comparison of count data $(n, \%)$ is verified by $\mathrm{X}^{2}$ value. When expressed as $\mathrm{P}<0.05$, it means that the comparison of this study is statistically significant ${ }^{[2]}$.

\section{Results}

\subsection{Therapeutic effect}

The effective number of patients in the experimental group was 48 , and the effective rate was $96.00 \%$. The effective number of patients in the control group was 41 , and the effective rate was $82.00 \%$. There is a big difference in the effective rate of treatment between the two groups, $\mathrm{P}<0.50$. There is statistical significance.

\begin{tabular}{ccccc}
\hline Group & Marked effective & Effective & Ineffective & Effective rate \\
\hline $\begin{array}{c}\text { Experimental Group } \\
(\mathrm{n}=50)\end{array}$ & $34(68.00)$ & $14(28.00)$ & $2(4.00)$ & $48(96.00)$ \\
Control Group $(\mathrm{n}=50)$ & $25(50.00)$ & $16(32.00)$ & $9(18.00)$ & $41(82.00)$ \\
$\mathrm{X}^{2}$ & & & $<0.05$ \\
$\mathrm{P}$ & & & \\
\hline
\end{tabular}

Figure 1 Comparison of curative effect between two groups of patients [n(\%)] 


\subsection{Vertebro-basal artery blood flow velocity changes}

Before treatment, there was no significant difference in the vertebral-basal artery blood flow velocity between the two groups of patients, $\mathrm{P}>0.05$. After the treatment intervention, the blood flow velocity all changed. In contrast, the vertebral and basilar artery blood flow velocity of the experimental group were higher than that of the control group, and the data between the groups were quite different, $\mathrm{P}<0.05$, which is significant.

\begin{tabular}{|c|c|c|c|c|}
\hline \multirow[b]{2}{*}{ Group } & \multicolumn{2}{|c|}{ Vertebral artery blood flow velocity } & \multicolumn{2}{|c|}{ Basilar artery blood flow velocity } \\
\hline & Before treatment & After treatment & Before treatment & After treatment \\
\hline $\begin{array}{l}\text { Experimental Group } \\
\qquad(\mathrm{n}=50)\end{array}$ & $20.34 \pm 5.44$ & $34.55 \pm 4.98$ & $22.34 \pm 4.95$ & $35.64 \pm 4.98$ \\
\hline $\begin{array}{l}\text { Control Group } \\
\quad(\mathrm{n}=50)\end{array}$ & $20.32 \pm 4.33$ & $30.23 \pm 3.76$ & $22.19 \pm 4.22$ & $30.23 \pm 3.65$ \\
\hline$X^{2}$ & 0.956 & 8.005 & 0.875 & 7.405 \\
\hline$P$ & $>0.05$ & $<0.05$ & $>0.05$ & $<0.05$ \\
\hline
\end{tabular}

Figure 2 Changes of vertebral-basal artery blood flow velocity before and after treatment in the two groups $\left(\bar{x}_{ \pm \mathrm{s}}\right)$

\subsection{Satisfaction}

The satisfaction degree in the experimental group was $94.00 \%$, and the satisfaction degree in the control group was $78.00 \%$. The data difference was statistically significant, and the satisfaction degree of the experimental group was higher.

\begin{tabular}{|c|c|c|c|c|}
\hline Group & Satisfactory & $\begin{array}{l}\text { Relatively } \\
\text { satisfactory }\end{array}$ & Unsatisfactory & Satisfaction degree \\
\hline $\begin{array}{l}\text { Experimental Group } \\
(\mathrm{n}=50)\end{array}$ & $35(70.00)$ & $12(24.00)$ & $3(6.00)$ & $47(94.00)$ \\
\hline $\begin{array}{c}\text { Control } \\
(\mathrm{n}=50)\end{array} \quad$ Group & $22(44.00)$ & $17(34.00)$ & $11(22.00)$ & $39(78.00)$ \\
\hline \multicolumn{5}{|l|}{$X^{2}$} \\
\hline $\mathrm{P}$ & & & & $<0.05$ \\
\hline
\end{tabular}

Figure 3 Comparison of treatment satisfaction between the two groups of patients $[\mathrm{n}(\%)]$

\section{Conclusion}

Cervical vertigo mainly refers to the disease of the extracranial segment of the vertebral artery, which leads to obstruction of the patient's blood flow, which manifests as vertigo syndrome. Judging from the current development situation in China, the incidence of cervical vertigo has shown an upward trend year by year. Patients with cervical vertigo are usually accompanied by migraine, restricted cervical spine movement, tinnitus, neck pain, soreness and stiffness, which is a serious threat to the patient's quality of life and life health. From the perspective of Chinese medicine, it is believed that cervical vertigo belongs to the scope of stroke, dizziness, headache etc, and is related to factors such as phlegm, deficiency, wind, and blood stasis. The main manifestations of dizziness in patients are phlegm and blood stasis mutual accumulation, Yang deficiency, middle Jiao blockage, no essence in blood, and thus leads to dizziness ${ }^{[3]}$. The treatment of phlegm and blood stasis and heat dissipating is the focus of the treatment of phlegm-obstruction vertigo. Jianpi Huatan Dingxuan Decoction is mainly derived from Banxia Baizhu Tianma Decoction and Xuanfu Daizhe Decoction. The prescription of Fuling and Alisma has the effects of invigorating the spleen and diuresis and eliminating phlegm, while Pinellia can invigorate the spleen, dry dampness and reduce phlegm; gastrodia elata has the effect of dissolving phlegm, dispelling wind, relieving dizziness, atractylodes yibaizhu mainly enhances the effect of drying dampness and expelling phlegm, which has the effect of taking its fragrance, avoiding 
obstruction and opening stagnation, inula is warm in nature, and can eliminate qi Phlegm, lowering the inverse; ochre is good at relieving and reversing, and has the effect of calming the liver and suppressing the yang, which can realize the effective treatment of vertigo with hyperactive liver yang. When the drugs are used in combination, they have the effects of invigorating the spleen, resolving phlegm, relieving dizziness and vomiting, and the effective rate is significant ${ }^{[4]}$. After the application of this drug in this study, the results showed that the treatment efficiency of patients in the experimental group was higher, as high as $96.00 \%$.

Modern medicine shows that insufficient blood supply to the vertebral-basal artery is the main factor leading to cervical vertigo. The increase in blood viscosity, hardening of the cerebral arteries, cervical spondylosis and other factors may also lead to insufficient blood supply to the vertebral-basal artery ${ }^{[5]}$. After transcranial Doppler ultrasound is given, the hemodynamics of the skull base arteries can be detected. The blood flow velocity of the vertebral basilar artery is the main content. It can respond to the compliance of the blood vessel and vascular resistance, which showed as a negative correlation ${ }^{[6]}$. According to clinical data, flunarizine hydrochloride can improve the symptoms of vertigo, but the effect of improving the accompanying symptoms is not obvious. After the application of Jianpi Huatan Dingxuan Decoction in this study, the results showed that the vertebrobasilar blood flow velocity of the experimental group of patients changed significantly, indicating that its application effect is better.

To sum up, in the treatment of patients with vertigo and phlegm obstruction, the application of Jianpi Huatan Dingxuan Decoction has a significant effect, which can promote the improvement of patients' clinical symptoms, and has positive significance for clinical development, it should be widely used.

\section{Acknowledgement}

At the completion of this thesis, I would like to thank all the people who helped with the writing. There are still some shortcomings in my writing of this thesis. I will continue to improve my future work to improve my own quality level, strengthen my research ability, solving academic problems and make in-depth supplements and keep strict demands on myself.

\section{References:}

1. Xueling Yan. Observation on the curative effect of Jianpi Huatan Dingxuan Decoction in the treatment of vertigo and obstructive phlegm syndrome [J]. Sichuan Traditional Chinese Medicine, 2011, 29(08): 60-61.

2. Qi Li. Evaluation of clinical efficacy and quality of life of circulatory ischemic vertigo after treatment with Huatan Dingxuan Decoction (phlegm obstruction syndrome) [D]. Changchun University of Traditional Chinese Medicine, 2017.
3. Chunhua Gu. Observation on the clinical efficacy of Jianpi Pinggan Decoction in the treatment of vertigo due to liver depression and spleen deficiency [D]. Guangzhou University of Traditional Chinese Medicine, 2019.

4. Xiaolin Wang, Wanqun Wang, Xue Deng, Xiaoyan Yan. Observation on the clinical efficacy of Qige Dingxuan Decoction in the treatment of vertebral base blood supply insufficiency and vertigo[J]. Clinical Research of Traditional Chinese Medicine, 2014, 6(24): 37-39.

5. Jun Ji, Xiuzhi Liu, Meng Li. Analysis of Dingxuan Decoction in the treatment of 32 cases of vertigo[J]. Shandong Medicine, 2015(12): 69.

6. Sheng Yang. A report of 45 cases of vertigo treated by Dingxuan Decoction[J]. Gansu Journal of Traditional Chinese Medicine, 2012(05): 21. 